\title{
Centrais de triagem de resíduos (CTR): uma solução para o gerenciamento em municípios de pequeno porte
}

\author{
Central sorting waste (CSW): a solution for the management in small cities \\ Juliana Penteado Coelho'; Marta Regina Lopes Tocchetto²; Erny Lauro Meinhardt Júnior ${ }^{3}$ \\ 'Departamento de Microbiologia - ICBS - UFRGS - POA - RS - Brasil \\ ${ }^{2}$ Departamento de Química - CCNE - UFSM - Santa Maria - RS - Brasil \\ ${ }^{3}$ Técnico da FEPAM - Fundação Estadual de Proteção Ambiental Henrique Luiz Roessler - RS - Brasil
}

\begin{abstract}
Resumo
No Brasil, os resíduos sólidos urbanos (RSU) ainda são depositados em locais irregulares. Algumas estratégias de gerenciamento podem mudar este cenário, como a redução da geração e a reciclagem de materiais, com a consequente disposição em aterro apenas do rejeito. A construção e o gerenciamento de aterros por municípios de pequeno porte é sempre difícil por apresentarem limitações financeiras e técnicas. As Centrais de Triagem de Resíduos (CTR) se constituem em uma solução viável considerando a possibilidade de melhorar a segregação e valorização dos materiais. O material triado é encaminhado à reciclagem ou à disposição em aterro. Esta solução pode ser consorciada, possibilidade regulamentada na Política Nacional dos Resíduos Sólidos, Lei 12.305 (BRASIL, 2010). O contexto apresentado motivou o presente trabalho que foi realizado em parceria, UFSM e FEPAM Regional Santa Maria/GERSEN. O objetivo traçado foi desenvolver um estudo para viabilizar a implantação de CTR em municípios de pequeno porte. Para conhecermos o perfil de geração dos pequenos municípios foram analisadas duas regiões localizadas no centro do Estado do Rio Grande do Sul. Cada uma dessas regiões foi composta por seis municípios. Os resultados demonstraram que o resíduo oriundo das áreas rurais é essencialmente reciclável, pois a fração orgânica que é separada na origem é reaproveitada e utilizada para produção de adubo ou é misturada à alimentação de animais. Assim, a parcela coletada e encaminhada à central de triagem apresenta alto aproveitamento, considerando posterior envio às empresas de reciclagem. Por outro lado, o resíduo gerado pela população urbana carece de uma melhor separação, a fim de reduzir o desperdício de materiais enviados para aterro. Este estudo permite afirmar que a implantação de CTR reduz os custos de gerenciamento de resíduos para os municípios, especialmente os de pequeno porte, pois é uma oportunidade de agregar valor à fração coletada e reduzir o volume a ser encaminhado ao aterro. A organização do setor a partir das Centrais, possibilita a implantação de programas de coleta seletiva e de educação ambiental, além do atendimento da Política Nacional de Resíduos Sólidos.
\end{abstract}

Palavras-chave: centrais de resíduos; municípios pequeno porte; gestão resíduos urbanos

\begin{abstract}
In Brazil, the solid urban waste (SUW) is still deposited in irregular places. Some management strategies can change this scenario, as the reduction in the generation and recycling of materials, with the consequent disposing in landfill only the tailing. Small cities present financial and technical difficulties in constructing and managing landfills. The central sorting waste (CSW) constitutes a viable solution considering the possibility to improve the segregation and evaluation of materials. The sorted material is forwarded to recycling or disposal in landfill. According to the National Solid Waste Politics, Law 12,305 (BRAZIL, 2010) this solution can be associated. The presented context motivated the current work which was conducted in partnership between UFSM and FEPAM Santa Maria Region/GERSEN. The objective was to develop a study to become viable the establishment of CSW in small cities. Two regions located in the center of Rio Grande do Sul State were analyzed in order to know the generation profile of small cities. Each of the regions was composed of six cities. The results showed that the residue derived from rural areas is essentially recyclable, since the organic fraction which is separated at source it is used to produce compost or it is mixed with animal feed. Thus, the portion collected and forwarded to the central sorting presents high utilization considering the later shipment to recycling companies. On the other hand, the residues generated by the urban population lacks of an appropriate separation, in order to reduce the waste of materials sent to landfill. This study allows us to state that the implementation of CSW reduces the municipal costs of waste management, especially for small cities, because it is an opportunity to add value to the collected fraction and to reduce the volume that would be sent to landfill. The organization of the sector from Centrals enables the implementation of selective collection programs and environmental education, as well as attending the National Policy on Solid Waste.
\end{abstract}

Keywords: solid urban waste, Small cities, municipal waste management. 


\section{INTRODUÇÃO}

O resíduo sólido urbano é um fenômeno inevitável e inesgotável, constituindo um dos grandes problemas na sociedade contemporânea, pois o acelerado aumento populacional e as crescentes necessidades de consumo geram um acúmulo significativo de lixo. O acúmulo de lixo urbano tem gerado, por exemplo, doenças devido às deficiências de saneamento ambiental nas cidades brasileiras; também é um dos fatores que contribui para o agravamento do efeito das enchentes, pois devido ao descarte incorreto provoca o entupimento dos bueiros. Os resíduos sólidos urbanos, em cerca de 42\% dos municípios brasileiros, são destinados diretamente para lixões (ABELPRE, 2012). Este índice revela a precariedade da situação, que exige uma tomada de decisão urgente, especialmente por parte dos gestores municipais. O custo para implantação de aterros urbanos e de coleta seletiva é elevado. A redução destes custos é possível a partir do gerenciamento integrado dos resíduos, realizado pelas associações de municípios. Esta alternativa é de especial interesse para as cidades de pequeno porte, que neste trabalho referem-se às que possuem até 20 mil habitantes (CRIVELARO et al, 2007). A implantação de centrais de triagem e/ou classificação e compostagem é uma alternativa intermediária a um sistema completo de gerenciamento, pois reduz, principalmente, os custos de disposição. Se houver associação entre municípios próximos, os custos se reduzem ainda mais, pois há uma divisão das despesas, além de um menor volume de rejeitos para transportar para o aterro. Soma-se às vantagens, a possibilidade dos resíduos selecionados serem enviados para a reciclagem e, assim gerarem receita, ainda que pequena. Outra vantagem é o atendimento dos princípios da Política Nacional de Resíduos Sólidos (PNRS) - Lei 12.305 - a qual estabelece que os municípios devem ter um plano de gestão e também desenvolver atividades de educação ambiental; realizar diagnóstico da situação dos resíduos sólidos; implantar programas para incentivar a criação e/ ou desenvolvimento de associações de catadores; possuir metas de redução, reutilização, coleta seletiva e reciclagem; dentre outras. O cumprimento da referida Lei nem sempre é uma tarefa fácil, especialmente para os de pequeno porte. Assim, a própria legislação flexibiliza a solução, a partir do estabelecimento de consórcios municipais. $\mathrm{O}$ art. 11 menciona esta possibilidade afirmando que os estados devem apoiar e priorizar as iniciativas de soluções consorciadas ou compartilhadas entre dois ou mais municípios. $\mathrm{O}$ incentivo à constru- ção destas parcerias pode ser observado no Art. 18 que afirma: os municípios que optarem por um consórcio serão priorizados na obtenção de recursos da União para desenvolverem ações com este objetivo (BRASIL, 2010). Todas estas ações também se refletem na melhoria da qualidade de vida das comunidades e do meio ambiente. O contexto apresentado determinou o estabelecimento do objetivo do presente trabalho: desenvolver estudo para viabilizar a implantação de centrais de triagem de resíduos (CTR) em municípios de pequeno porte. A realização deste estudo permite afirmar que a implantação de CTR reduz os custos de gerenciamento de resíduos para os municípios, especialmente para os de pequeno porte, pois é uma oportunidade de agregar valor à fração coletada e reduzir o volume a ser encaminhado para aterro. A organização do setor, a partir das Centrais possibilita também a implantação de programas de coleta seletiva e de educação ambiental, além do atendimento da Política Nacional de Resíduos Sólidos. A implantação destas unidades pode ser replicada em qualquer município do estado do Rio Grande do Sul ou do Brasil.

\section{FUNDAMENTAÇÃO TEÓRICA}

\section{I Geração dos Resíduos}

A realidade nos grandes centros urbanos é que o lixo cresceu em quantidade e diversidade, a ponto de exigir urgentemente uma conscientização por parte da sociedade, do poder público e do setor privado, no sentido de reduzi-lo, modificá-lo e tratá-lo. O estudo denominado Panorama dos Resíduos Sólidos no Brasil demonstra que cada brasileiro gera, em média, $1 \mathrm{~kg}$ de lixo por dia (ABELPRE, 2012). Dependendo da região e do poder aquisitivo este volume pode chegar a níveis ainda maiores. O perfil da geração do lixo no Brasil há um tempo era de procedência orgânica, nos últimos anos este contorno vem acompanhando o modo de consumo dos países ricos, ou seja, levando a uma intensificação do uso de produtos descartáveis (FADINI e FADINI, 2001). Esta mudança de perfil influencia na escolha das estratégias de gerenciamento e na destinação a ser dada a estes resíduos.

\subsection{Destinação de Resíduos Urbanos}

O destino final do RSU em lixão é uma forma inadequada de disposição e se caracteriza pela simples descarga sobre o solo, sem medidas de proteção ao meio ambiente e à saúde pública (CITTADIN, 2006). O local adequado para a 
destinação final dos rejeitos urbanos é o aterro. Esta é uma técnica de disposição, cujos danos à saúde e os riscos relacionados à sua segurança causados pelos impactos decorrentes do descarte direto no solo, são minimizados. Aterros são obras de engenharia que se destinam ao confinamento seguro dos resíduos sólidos em uma área previamente licenciada. A destinação correta, no Brasil corresponde a aproximadamente $42 \%$ do volume de resíduos coletado (ABELPRE, 2012). Este valor demonstra que o país ainda precisa evoluir no gerenciamento, tendo em vista a pretensão da irradicação dos lixões até 2014 (BRASIL, 2010). As Centrais de Triagem podem contribuir muito neste sentido.

\subsection{Centrais de Triagem de Resíduos}

As Centrais de Triagem de Resíduos (CTR) são locais onde é realizada a segregação dos mesmos após o recolhimento pelo serviço de coleta. Uma melhor separação pode ser realizada nas Centrais de Triagem e Compostagem de resíduos (CTCR) são centros de separação das frações inorgânicas e orgânicas dos resíduos sólidos urbanos, operacionalizadas em maior ou menor escala por equipamentos eletromecânicos (SCHALCH et al, 2002). Também podem ser operacionalizadas de forma manual. Nestas unidades, a separação dos resíduos orgânicos destina-se à compostagem. Em consequência disso, tem-se uma menor quantidade de resíduos sendo encaminhada aos aterros. As centrais de triagem, associadas à coleta seletiva, têm grande importância preliminar à reciclagem, pois permitem uma melhor separação e comercialização dos materiais, que posteriormente serão reprocessados e/ou reincorporados aos processos produtivos. BARON (2010) menciona que desta maneira, podemos dizer que as unidades de triagem prestam grande contribuição à sociedade $\mathrm{e}$ ao meio ambiente, pois além de aumentar a vida útil dos aterros e promover a inclusão social dos trabalhadores envolvidos, ajudam na economia de recursos naturais. A implantação de central de triagem deve ser entendida como uma etapa intermediária do sistema integrado de gerenciamento dos resíduos sólidos.

No presente trabalho diferencia-se central de triagem de central de classificação de resíduos. Central de triagem considera que a seleção é executada em resíduos cuja segregação pelo gerador é ausente ou precária. No caso de centrais de classificação, considera-se que o gerador tenha realizado uma separação mais criteriosa das frações inorgânica e orgânica do resíduo. Esta diferença conceitual está relacionada também com o gerenciamento da unidade, tendo em vista que nas centrais de classificação obtém-se um percentual maior de recicláveis, diferentemente do que ocorre nas centrais de triagem, nas quais os resíduos apresentam contaminação e misturas que podem inviabilizar o reaproveitamento. Esta diferenciação não se verifica nos trabalhos e publicações da área, porém entende-se que ela é importante e deve ser adotada porque não é se relaciona apenas aos conceitos, mas implica em aspectos construtivos, gerenciais e ambientais das áreas destinas a centrais de resíduos. As Centrais de Triagem e de Classificação podem existir independentemente do sistema de compostagem. Naturalmente que a associação destas estratégias, ao contemplar as duas frações principais que compõem os resíduos urbanos (orgânica e inorgânica), traz vantagens significativas ao sistema de gerenciamento. Porém, entende-se que a implantação pode ser gradual, ou seja, primeiramente a Central de Triagem, e à medida que segregação for melhorando é possível implantar na área, espaço para realização de compostagem e, inclusive o beneficiamento de outros resíduos, como, por exemplo, os da construção civil.

\section{METODOLOGIA}

O estabelecimento do perfil da geração de resíduos de pequenos municípios pertencentes à Região Central do Rio Grande do Sul foi possível a partir da análise e interpretação dos dados obtidos a partir de um questionário aplicado pela FEPAM/GERCEN. A análise teve como objetivo diagnosticar a situação destes municípios, em relação à geração e ao gerenciamento dos resíduos sólidos. Ainda, foi realizado acompanhamento de visitas realizadas pela equipe da FEPAM a estas localidades, a fim de obter maiores informações e detalhamentos sobre as respostas fornecidas e que eram de interesse ao presente trabalho. $\mathrm{O}$ estudo foi desenvolvido no período compreendido de janeiro de 2011 a agosto de 2012.

Os municípios escolhidos localizam-se próximos à cidade de Santa Maria (RS), que é a quinta maior cidade do estado do Rio Grande do Sul e possui 261.027 habitantes (FAMURS, 2012). O agrupamento foi estabelecido em duas regiões (Região 1 e Região 2), tendo como base a possibilidade do gerenciamento integrado dos resíduos, a partir da implantação de uma CTR em um dos municípios. A escolha do município para a implantação da CTR considerou a facilidade de acesso às demais localidades da respectiva região. A Figura 1 relaciona os municípios que compõe 


\begin{tabular}{|c|c|c|c|c|c|}
\hline $\begin{array}{l}\text { REGIÃO } 1 \\
\text { CIDADES }\end{array}$ & $\begin{array}{l}\text { POPULAÇÃO } \\
\text { ( } \mathrm{n}^{\circ} \text { de hab.) }\end{array}$ & $\begin{array}{l}\text { POPULAÇÃO } \\
\text { URBANA } \\
\text { (nº de hab.) }\end{array}$ & $\begin{array}{l}\text { POPULAÇÃO } \\
\text { RURAL } \\
\text { (n } \mathrm{n}^{\circ} \text { de hab.) }\end{array}$ & $\begin{array}{c}\text { RELAÇÃO } \\
\text { POPULAÇÃO } \\
\text { URBANA E TOTAL } \\
(\%)\end{array}$ & $\begin{array}{c}\text { RELAÇÃO } \\
\text { POPULAÇÃO } \\
\text { RURAL } \\
\text { E TOTAL } \\
(\%)\end{array}$ \\
\hline Agudo & 16.729 & 6.894 & 9.835 & 41,2 & 58,8 \\
\hline Dona Francisca & 3.401 & 2.146 & 1.255 & 63,1 & 36,9 \\
\hline Faxinal do Soturno & 6.672 & 4.175 & 2.497 & 62,6 & 37,4 \\
\hline Nova Palma & 6.345 & 3.083 & 3.262 & 48,6 & 51,4 \\
\hline Restinga Seca & 15.850 & 8.982 & 6.868 & 56,7 & 43,3 \\
\hline São João do Polêsine & 2.635 & 1.354 & 1.281 & 51,4 & 48,6 \\
\hline Total & 51.632 & 26.634 & 24.998 & & \\
\hline $\begin{array}{l}\text { REGIÃO } 2 \\
\text { CIDADES }\end{array}$ & $\begin{array}{l}\text { POPULAÇÃO } \\
\text { (n }{ }^{\circ} \text { de hab.) }\end{array}$ & $\begin{array}{l}\text { POPULAÇÃO } \\
\text { URBANA } \\
\text { (nº de hab.) }\end{array}$ & $\begin{array}{l}\text { POPULAÇÃO } \\
\text { RURAL } \\
\text { (nº de hab.) }\end{array}$ & $\begin{array}{c}\text { RELAÇÃO } \\
\text { POPULAÇÃO } \\
\text { URBANA E TOTAL } \\
(\%)\end{array}$ & $\begin{array}{c}\text { RELAÇÃO } \\
\text { POPULAÇÃO } \\
\text { RURAL } \\
\text { E TOTAL } \\
(\%) \\
\end{array}$ \\
\hline Dilermando de Aguiar & 2.588 & 991 & 2.073 & 32,3 & 67,7 \\
\hline Jari & 3.575 & 613 & 2.962 & 17,1 & 82,9 \\
\hline Mata & 5.111 & 2.618 & 2.493 & 51,2 & 48,8 \\
\hline Quevedos & 2.710 & 852 & 1.858 & 31,4 & 68,6 \\
\hline São Pedro do Sul & 16.371 & 11.932 & 4.439 & 72,9 & 27,1 \\
\hline Toropi & 2.952 & 611 & 2.341 & 20,7 & 79,3 \\
\hline Total & 33.307 & 17617 & 16.166 & & \\
\hline
\end{tabular}

Figura 1 - Número de Habitantes nas Zonas Urbana e Rural nas Regiões do estudo e as respectivas relações percentuais Fonte: Federação das Associações de Municípios do Rio Grande do Sul (FAMURS)

as Regiões do estudo, 1 e 2, com as respectivas populações urbana e rural. Também se apresenta a população total de cada um dos municípios e as relações percentuais entre população urbana e rural e vice versa.

As estimativas apresentadas no trabalho sobre a quantidade de RSU gerada pelos municípios foram realizadas após a análise dos dados do questionário, as visitas e os contatos com os responsáveis pelo setor ambiental. Considerouse como base que cada habitante produz $1 \mathrm{~kg} /$ dia, como já mencionado anteriormente (média nacional).

As informações e os dados obtidos serviram para subsidiar a proposta de gerenciamento que consiste na implantação de centrais de triagem de resíduos fundamentadas na estruturação de um sistema de coleta seletiva que futuramente, podem evoluir para classificação/compostagem. Esta proposta tanto pode ser implementada por consórcio dos municípios que compõe as regiões do estudo ou por qualquer outro grupo de locali- dades que tenha interesse.

\section{RESULTADOS E DISCUSSÕES}

As Figuras 2 e 3 apresentam a geração mensal de resíduos em cada um dos municípios estudados, considerando as Regiões 1 e 2. Observando as informações percebe-se que em alguns meses, não há registro da quantidade coletada. É o caso de Nova Palma nos meses de novembro e dezembro de 2011. Este fato foi detectado como uma realidade comum no grupo em estudo. Essa falta de controle de geração traz dificuldades para a implantação de um sistema de gerenciamento que vise minimizar a quantidade gerada.

Observam-se na Figura 2 alguns resultados discrepantes, como em São João do Polêsine. Nos meses de novembro e dezembro de 2011, a quantidade coletada foi acima de 80 toneladas, por outro lado nos meses seguintes, o volume diminuiu de forma expressiva, chegando ao mês de março a 


\begin{tabular}{|c|c|c|c|c|c|c|}
\hline $\begin{array}{l}\text { Região } 1 \\
\text { Meses }\end{array}$ & nov/11 & $\mathrm{dez} / 11$ & $\mathrm{jan} / 12$ & $\mathrm{fev} / 12$ & $\mathrm{mar} / 12$ & $\mathrm{abr} / 12$ \\
\hline $\begin{array}{l}\text { AGUDO } \\
(\mathrm{kg})\end{array}$ & 108080 & 132680 & 128190 & 108550 & 110760 & 113700 \\
\hline $\begin{array}{l}\text { DONA FRANCISCA } \\
(\mathrm{kg})\end{array}$ & 24420 & 28790 & 27850 & 28070 & 24220 & 26830 \\
\hline $\begin{array}{l}\text { FAXINAL DO SOTURNO } \\
\qquad(\mathrm{kg})\end{array}$ & 85340 & 124790 & 88050 & 71230 & 101920 & 70420 \\
\hline $\begin{array}{l}\text { NOVA PALMA } \\
(\mathrm{kg})\end{array}$ & $\mathrm{x}$ & $x$ & 42430 & 49450 & 7480 & $\mathrm{x}$ \\
\hline $\begin{array}{c}\text { SÃO JOÃO } \\
\text { DO POLÊSINE } \\
(\mathrm{kg})\end{array}$ & 84160 & 85790 & 28220 & 19350 & 11390 & $\mathrm{x}$ \\
\hline $\begin{array}{l}\text { RESTINGA SECA } \\
(\mathrm{kg})\end{array}$ & 30610 & 85850 & 87690 & 102610 & 79540 & 57900 \\
\hline $\begin{array}{c}\text { QUANTIDADE } \\
\text { TOTAL COLETADA } \\
(\mathbf{k g})\end{array}$ & 332610 & 457900 & 402430 & 379260 & 335310 & 268850 \\
\hline
\end{tabular}

Figura 2 - Quantidade mensal de resíduos coletada nos municípios da Região 1

Fonte: FEPAM/GERCEN

\begin{tabular}{|c|c|c|c|c|c|c|}
\hline Região 2 & mar/11 & $\mathrm{abr} / 11$ & mai/11 & jun/11 & jul/11 & ago/11 \\
\hline $\begin{array}{l}\text { DILERMANDO DE AGUIAR } \\
\qquad(\mathrm{kg})\end{array}$ & 8580 & 7650 & 7680 & 8950 & 3730 & 17680 \\
\hline JARI (kg) & 750 & 3880 & $\mathrm{x}$ & $\mathrm{x}$ & $\mathrm{x}$ & $\mathrm{x}$ \\
\hline MATA (kg) & 36570 & 43980 & 48460 & 46860 & 41420 & 51800 \\
\hline QUEVEDOS (kg) & $\mathrm{x}$ & $\mathrm{x}$ & $\mathrm{x}$ & 16840 & 192930 & 171480 \\
\hline SÃO PEDRO DO SUL $\quad(\mathrm{kg})$ & 174840 & 171760 & 173800 & 170740 & 169260 & 183070 \\
\hline TOROPI (kg) & 17980 & 11820 & 10080 & $\mathrm{x}$ & $\mathrm{x}$ & $\mathrm{x}$ \\
\hline $\begin{array}{l}\text { QUANTIDADE TOTAL COLETADA } \\
(\mathbf{k g})\end{array}$ & 238720 & 239090 & 240020 & 243390 & 407340 & 424030 \\
\hline
\end{tabular}

Figura 3 - Quantidade mensal de resíduos coletada nos municípios da Região 2 onte: FEPAM/GERCEN 
menos de 12 toneladas/mês. Os motivos para essa discrepância podem ser: controle deficiente das informações enviadas pela prefeitura; evidência de desperdício nos meses com valores maiores; coleta deficiente nos meses em que se verifica uma significativa redução; somatório das quantidades que compõem a carga, pois um mesmo caminhão leva resíduos de mais de um município, tendo em vista a terceirização do transporte. Outros motivos também podem explicar esta diferença: sazonalidade, festividades regionais e/ou período de férias. Estes fatores exercem influência expressiva na geração de resíduos em determinados períodos do ano.

Deve-se salientar a diferença existente em relação à coleta dos resíduos gerados pelas populações urbana e rural. A população urbana tem seu RSU coletado, praticamente, diariamente. Outra característica que distingue as diferentes zonas em um mesmo município, é que os resíduos oriundos da zona urbana, normalmente, encontram-se misturados. Por outro lado, o resíduo gerado pela população rural é coletado com menor frequência e também em bem menor a quantidade. Isso se deve a um maior reaproveitamento como, por exemplo, os restos de alimentos são destinados aos animais ou são incorporados ao solo para produção de adubo. Assim, a fração orgânica é praticamente inexistente para destinação ao aterro. Portanto, os resíduos coletados das áreas rurais são excelentes para serem enviados para uma central de triagem, pois apresentam uma grande proporção de recicláveis e uma baixa contaminação com resíduos orgânicos. Esta característica é importante para agregar valor aos resíduos enviados à reciclagem.

Com a melhoria da triagem nas centrais, aumenta-se a recuperação nas centrais. Um índice de $10 \%$ de material recuperado na triagem representa uma expressiva recuperação em relação à quantidade coletada. Estima-se que com a implantação da coleta seletiva associada à CTR, o percentual poderá chegar a $25 \%$ de recuperação. Os materiais recuperados podem representar fonte de renda para os catadores associados ao sistema. Também significa economia de transporte em virtude de menor quantidade a ser destinada ao aterro, assim como aumento da vida útil destas áreas e redução dos custos de gerenciamento. A triagem de materiais também traz benefícios ambientais, pois reduz a extração de recursos naturais que, muitas vezes não são renováveis, como o petróleo.

\section{CONCLUSÃO}

Com base nas informações e dados obtidos pelo estudo e pelas visitas realizadas, o município de São João do Polêsine apresenta as condições adequadas para a implantação de uma central de triagem de resíduos para atender a Região 1. Para a Região 2, Dilermando de Aguiar demonstra possuir as condições ideais para abrigar a CTR. Os municípios foram escolhidos por apresentarem facilidade de acesso para os demais que compõem as referidas regiões.

A economia financeira com a formação de associações para a implantação de Centrais de Triagem de Resíduos, ou seja, o que deixa de ser gasto individualmente para dispor de resíduo em aterro, pode ser revertido em benefícios socioambientais tanto para os envolvidos diretamente no gerenciamento quanto para a população como um todo. É preciso também considerar alguns valores intangíveis para a economia tradicional, como o bem estar e menores riscos às comunidades, em virtude de uma menor quantidade de lixo disposto; um melhor aproveitamento de áreas, pois evita o sacrifício das mesmas para "enterrar" lixo; uma menor exploração de recursos naturais, tendo em vista o encaminhamento de uma fração de resíduos para a reciclagem; melhoria da qualidade de vida das populações devido à internalização de valores socioambientais; maior consciência e estímulo à educação ambiental; ajuste da gestão dos resíduos nos municípios à PNRS; dentre outros.

Os catadores são vitais em uma central de triagem, portanto uma parte dos ganhos obtidos com o aumento do percentual de resíduo encaminhado à reciclagem e/ou com o aumento do valor agregado na comercialização dos mesmos, poderia ser revertida em "bônus de seletividade" nos moldes dos estabelecidos para premiar a produtividade. A melhoria dos rendimentos se reverteria em melhores condições de vida para si e para a sua família.

O gerenciamento dos RSU depende muito da mudança dos hábitos da população, pois é de fundamental importância o reconhecimento da responsabilidade individual para que este ocorra de forma adequada. É importante também que os gestores municipais apoiem a implantação de centrais de triagem de resíduos associada às demais estratégia de gerenciamento, pois requer investimentos relativamente pequenos, ainda mais considerando a possibilidade de implantação consorciada. Como vantagem imediata pode-se apontar a redução dos gastos de gerenciamento. A implantação de uma central sugere-se partir da iniciativa pública, mas nada impede que a iniciativa privada incorpore esta ideia e/ou colabore para a concretização da mesma. 


\section{REFERÊNCIAS}

ABELPRE. Associação Brasileira de Empresas de Limpeza Pública e Resíduos Especiais. Panorama dos Resíduos Sólidos no Brasil. São Paulo, 2012

BARON, Viviane. Licenciamento Ambiental de Centrais de Triagem de Resíduo Sólido Urbano. Relatório da disciplina de Laboratório de Arquitetura e Urbanismo. Universidade de Caxias do Sul. Caxias do Sul, 2010.

BRASIL. Lei ${ }^{\circ} 12.305$, de 2 de agosto de 2010. Institui a Política Nacional de Resíduos Sólidos. Brasília, 2010.

CITTADIN, Leandro - Análise de critérios operacionais do aterro sanitário do Cirsures. Trabalho de Conclusão de Curso de Engenharia Ambiental UNESC. Criciúma, 2006.

CRIVELARO, Sérgio Henrique Rezende; SOUZA, Aline Tadei; FRANCISCO, José. Pequenos municípios: a participação do dia-a-dia e a cidadania do cara-a-cara. Anais do II Seminário Nacional: Movimentos Sociais, Participação e Democracia, Florianópolis, 2007.

FADINI, Pedro Sérgio e FADINI, Almerinda Antonia Barbosa. Lixo: desafios e compromissos. Caderno temático de Química Nova na Escola, 2001. Disponível em: < http://qnesc.sbq.org.br/ online/cadernos/01/lixo.pdf $>$. Acesso em: 02 mar. 2012.

FAMURS, Federação das Associações de Municípios do Rio Grande do Sul. Disponível em: <http:// www.famurs.com.br/> Acesso em: 02 fev. 2012.

SCHALCH, V; LEITE, W.C.A;FERNANDES, J.L.; CASTRO, M.C.A.A. Gestão e gerenciamento de resíduos Sólidos. São Carlos, 2002. 